\title{
The reflection of very cold neutrons from diamond powder nanoparticles
}

\author{
V. V. Nesvizhevsky \\ Institute Laue-Langevin, 6 rue Jules Horowitz, F-38042, Grenoble, France \\ E. V. Lychagin, A. Yu. Muzychka, A. V. Strelkov \\ Joint Institute for Nuclear Research, 141980, Dubna, Russia \\ G. Pignol, K.V. Protasov \\ LPSC, UJF - CNRS/IN2P3 - INPG, 53, rue des Martyrs, F-38026 Grenoble, \\ France
}

\begin{abstract}
We study possibility of efficient reflection of very cold neutrons (VCN) from powders of nanoparticles. In particular, we measured the scattering of VCN at a powder of diamond nanoparticles as a function of powder sample thickness, neutron velocity and scattering angle. We observed extremely intense scattering of VCN even off thin powder samples. This agrees qualitatively with the model of independent nanoparticles at rest. We show that this intense scattering would allow us to use nanoparticle powders very efficiently as the very first reflectors for neutrons with energies within a complete $\mathrm{VCN}$ range up to $10^{-4} \mathrm{eV}$.
\end{abstract}

Key words: 28.20.-v; 29.25.Dz; 78.90.+t

\section{Introduction}

In the present article we report on the feasibility of efficiently reflecting very cold neutrons (VCN) at a powder of diamond nanoparticles, thus bridging the energy gap between efficient reactor reflectors [1] for thermal and cold neutrons, and the effective Fermi potential for ultracold neutrons (UCN) [2,3,4].

Email address: nesvizhevsky@ill.fr (V. V. Nesvizhevsky). 
The use of nanoparticles provides a sufficiently large cross-section for coherent interaction and inhomogeneity of the moderator density on a spatial scale of about the neutron wavelength [5]. Nevertheless, many neutron-nanoparticle collisions are needed in order to reflect $\mathrm{VCN}$ as they scatter preferably to straightforward direction. A large number of collisions constrain the choice of materials: only low absorbing ones with high effective Fermi potential are appropriate. Thus, diamond nanoparticles is an evident candidate for such VCN reflector.

The formation of diamond particles by explosive shock was first observed more than forty years ago [6]. Since then very intensive studies of their production and of their various applications have been performed worldwide. These particles measure a few nanometers [7]. Nanoparticles consist of a diamond nucleus within an onion-like shell with a complex chemical composition [8]. A recent review of the synthesis, structure, properties and applications of diamond nanoparticles can be found in [9].

Scattering off nanoparticles could be used also to study the dynamics of nanoparticles, at a surface, in the material bulk [10,11]12], and even probably to cool VCN down to the UCN energy range [13,14,15]. On the other hand, other studies have shown that the quasi-elastic scattering of UCN at nanoparticles on solid surfaces could be responsible for false effects in storage experiments in fundamental particle physics [16,17, 18, 19,20].

The first experiments on the reflection of VCN from nanostructured materials as well as on VCN storage were carried out in the seventies in [21] and later continued in 22. We have extended the energy range and the efficiency of VCN reflection by exploiting diamond nanoparticles. A reflector of this type would be particularly useful for both UCN sources using ultracold nanoparticles [5]23] and for VCN sources; it would not be efficient however for cold and thermal neutrons, as shown in [24. We estimated the maximum energy of the reflected neutrons. We also explored the reliability of the theoretical predictions for the interaction of neutrons with nanoparticle powders, and we provide an overview of the phenomena relevant to the interaction.

In section II we describe the experimental installation developed for these studies, and the measuring procedure. In section III we describe the samples of ultradiamond powder used (so called ultradiamond90 [7]) and the procedures for measuring the intensity and the angular distribution of the scattered neutrons. In section IV we analyse the results, comparing them with Monte Carlo simulations based on a model of independent nanoparticles at rest. 


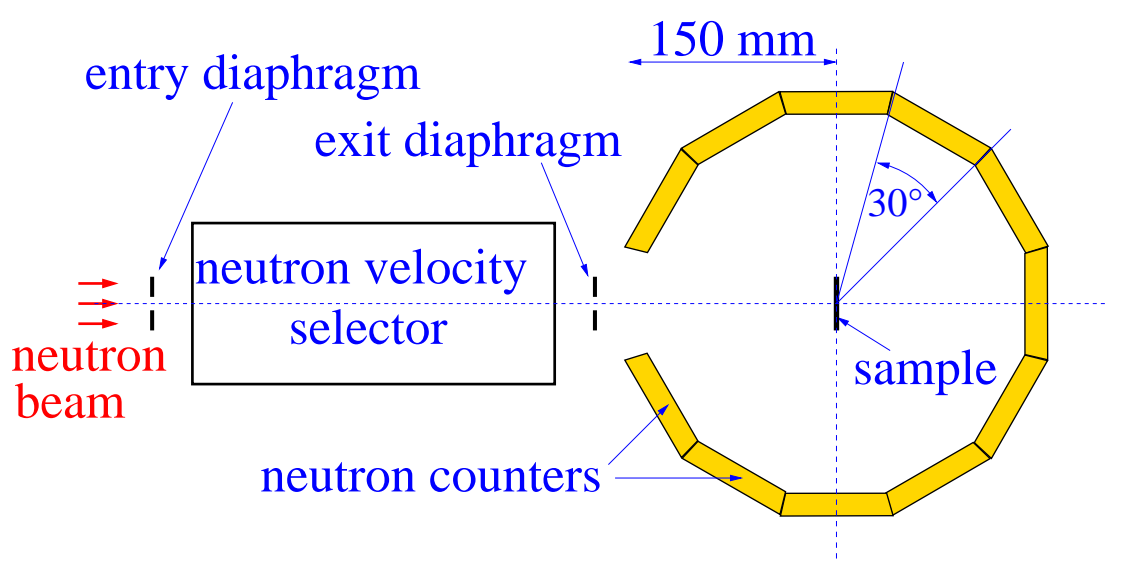

Fig. 1. The experimental setup (view from above)

\section{The experimental installation}

The setup is shown in fig. 1 (viewed from above). Two boron rubber diaphragms with a thickness of $5 \mathrm{~mm}$ shape the neutron beam; the beam axis is set in a horizontal plane. The diameter of the entrance diaphragm is $16 \mathrm{~mm}$; the diameter of the exit diaphragm is $10 \mathrm{~mm}$; the distance between the diaphragms is $116 \mathrm{~cm}$. A mechanical velocity selector is installed between them. A sample is placed at a distance of $23 \mathrm{~cm}$ downstream of the exit diaphragm in the middle of the detector assembly. The detector assembly consists of 11 neutron counters measuring the neutrons scattered by the sample. The counters' flat entrance windows form 11 sides of a regular 12-sided prism. The neutron beam enters through the remaining open $12^{\text {th }}$ side, perpendicular to the assembly. The counter directly opposite the entrance measures the neutrons passing through; its window is covered by a cadmium plate of thickness $0.5 \mathrm{~mm}$ and is pierced with a $3 \mathrm{~cm}$-diameter hole in the line of the beam. The other counters measure the neutrons scattered $30^{\circ}$ in a horizontal plane and $60^{\circ}$ in a vertical plane (the solid angle of $\pi / 6$ ). The detector assembly is surrounded by cadmium, borated polyethylene and boron rubber neutron shieldings. In order to decrease neutron scattering in the air we placed the detector assembly in a polyethylene bag filled with argon (Ar). The neutron entry window consisted of a flat quartz plate with a thickness of $3.5 \mathrm{~mm}$.

Each of the 11 proportional gaseous neutron counters has a thin aluminum window with a thickness of $200 \mu \mathrm{m}$. The counters are filled with a mixture of $\mathrm{Ar}$ and ${ }^{3} \mathrm{He}$ gas at a pressure of 1 bar. The ${ }^{3} \mathrm{He}$ partial pressure is equal to $130 \mathrm{mbar}$; the thickness of the gas-sensitive layer is $18 \mathrm{~mm}$. The detection efficiency for neutrons with a velocity of $100 \mathrm{~m} / \mathrm{s}$ is therefore equal to $\approx 50 \%$; the efficiency for neutrons with a velocity of $40 \mathrm{~m} / \mathrm{s}$ is equal to $\approx 85 \%$. It should be noted that absolute efficiency values are irrelevant when measuring neutron elastic scattering probabilities if all the counters are equally efficient. 
Finally, the neutron velocity selector has a resolution of $10-15 \%$ in the whole VCN velocity range.

\section{The experiment with diamond powder nanoparticles}

The measurement was carried out at the VCN PF2 beam position [25] at the Institut Laue-Langevin (Grenoble, France), providing access to neutrons with a velocity in the range of $30-160 \mathrm{~m} / \mathrm{s}$. Our sample was a powder of diamond nanoparticles (ultradiamond90 [7]) with diameters in the range of $2-5 \mathrm{~nm}$ and a known size distribution [7].

The powder was placed between two sapphire plates with a thickness of $1 \mathrm{~mm}$ each; the space between the plates was sealed on all sides with aluminum or Teflon foil. Once the powder was in position it was subjected to vibration to increase its density; the resulting density was equal to $0.6 \mathrm{~g} / \mathrm{cm}^{3}$. We prepared four samples with a thickness of $0.2,0.4,2$ and $6 \mathrm{~mm}$. The choice of thicknesses was determined as follows. The thinner the sample, the lower the multiple scattering effect. Thinner samples thus allow us to study neutronnanoparticle interactions. The thicker the sample, the larger the reflectivity. Thicker samples thus allow us to assess the feasability of using nanopowder as a reflector. The minimal thickness of a layer of this type, without significant holes, is $0.2 \mathrm{~mm}$.

Fig. 2 presents examples of the angular dependencies measured for neutrons scattered at a $2 \mathrm{~mm}$-thick sample of ultradiamond90.

In order to check the elasticity of the neutron scattering at the ultradiamond90 powder samples, we measured the time-of-flight spectrum of the scattered neutrons using a counter placed at an angle $150^{\circ}$. The average initial neutron velocity was equal to $60 \mathrm{~m} / \mathrm{s}$. The results of a comparison of the spectrum of scattered neutrons with the initial spectrum measured in analogous fashion are presented in fig. 3. To demonstrate the sensitivity of this method, we also measured the scattering of neutrons at a polyethylene sample with a thickness of $2 \mathrm{~mm}$. We were thus able to show that VCN scattering at the nanoparticles is mainly elastic.

\section{Analysis}

In order to analyse the data measured we developed a Monte Carlo model of the experiment. The interaction of the neutrons with a nanoparticle powder can be described using the simple approach proposed in [23]. We neglected 

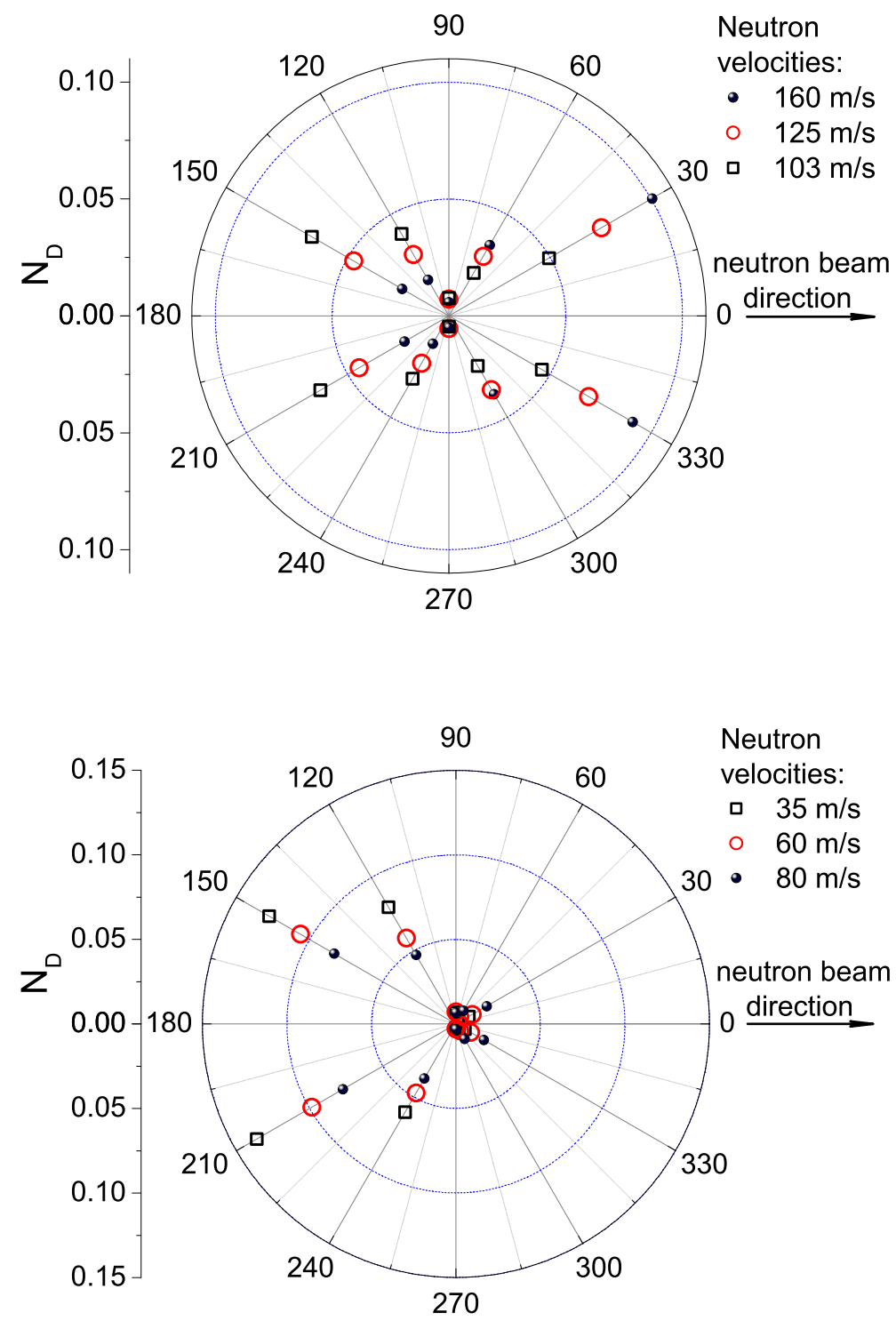

Fig. 2. The scattered neutron flux (normalized to the initial neutron flux) is shown as a function of the scattering angle for an ultradiamond90 powder sample with a thickness of $2 \mathrm{~mm}$. The angle measured is that between the incoming beam of neutrons and its line of travel from the sample to the center of a corresponding counter. The results for all measured velocities from $35 \mathrm{~m} / \mathrm{s}$ to $160 \mathrm{~m} / \mathrm{s}$ are indicated by the different types of point.

the relatively complex internal structure of the nanoparticle and modelled it as a uniform sphere. The neutron-nanoparticle elementary interaction was calculated using the first Born approximation. The absorption and elastic cross-sections, as well as the angular distribution of the scattered neutrons, were calculated analytically. 


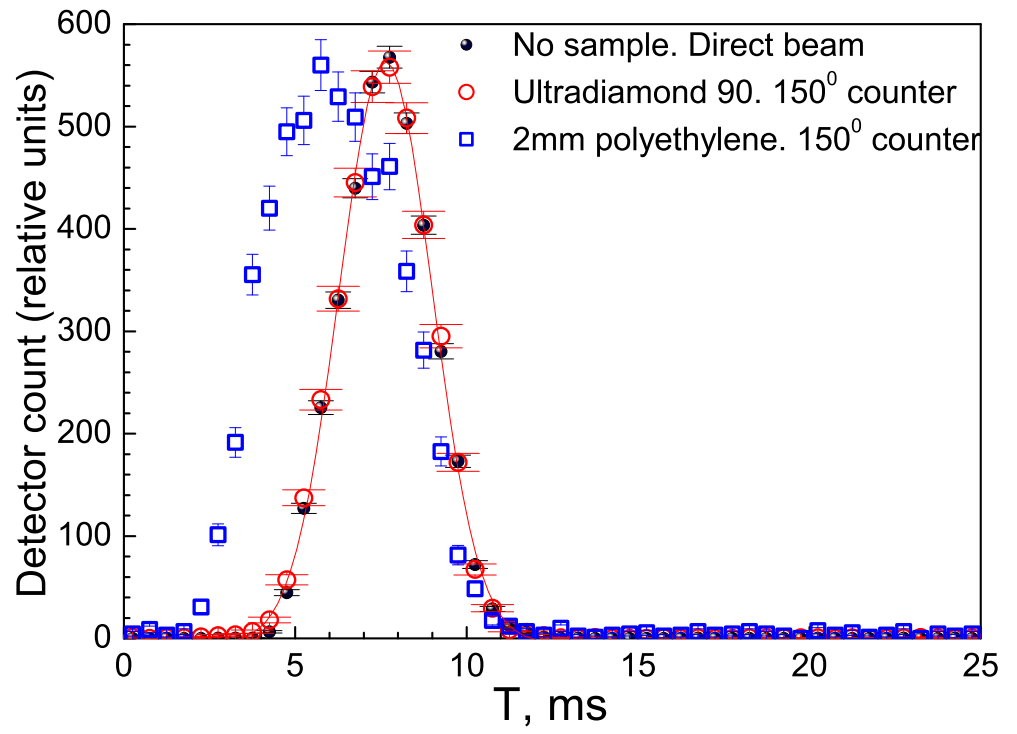

Fig. 3. The neutron count rate is presented as a function of the time of flight of the neutrons with an average initial velocity of $60 \mathrm{~m} / \mathrm{s}$. The black circles correspond to the initial neutron spectrum. The open circles indicate the data for the spectrum of neutrons scattered to an angle of $150^{\circ}$. The thickness of the ultradiamond90 powder sample is equal to $2 \mathrm{~mm}$. The squares show results for the scattering of neutrons at a polyethylene sample with a thickness of $2 \mathrm{~mm}$, measured at the same counter.

The chemical composition of the nanoparticle is complex and includes carbon (up to $88 \%$ ), hydrogen (1.0\%), nitrogen (2.5\%), oxygen (up to 10\%) [26]. Moreover, a certain amount of water covers a significant surface area of the nanoparticles. In general, the hydrogen in the water and on the surface of the nanoparticles scatters the neutrons up to the thermal energy range ("upscattering"); thermal neutrons do not interact as efficiently with nanoparticles and therefore traverse the powder. As the amount of hydrogen was not precisely known we considered it as a free parameter in the model.

We built a Monte Carlo model of the experiment on this basis, taking into account the sample and installation geometry described above. The size distribution of the nanoparticles in the powder is assumed to be equivalent to that presented in [7].

The simulation results are compared to the probabilities measured of forward and backward scattering of VCN at samples of all measured thicknesses as shown in fig. 4. The two sets of curves correspond to the calculation with pure carbon nanoparticles (solid lines) and to that with $1 \%$ of hydrogen added (dotted lines).

In the broad range of VCN velocities and sample thicknesses, good qualitative agreement was obtained between the probability and the angular distribu- 

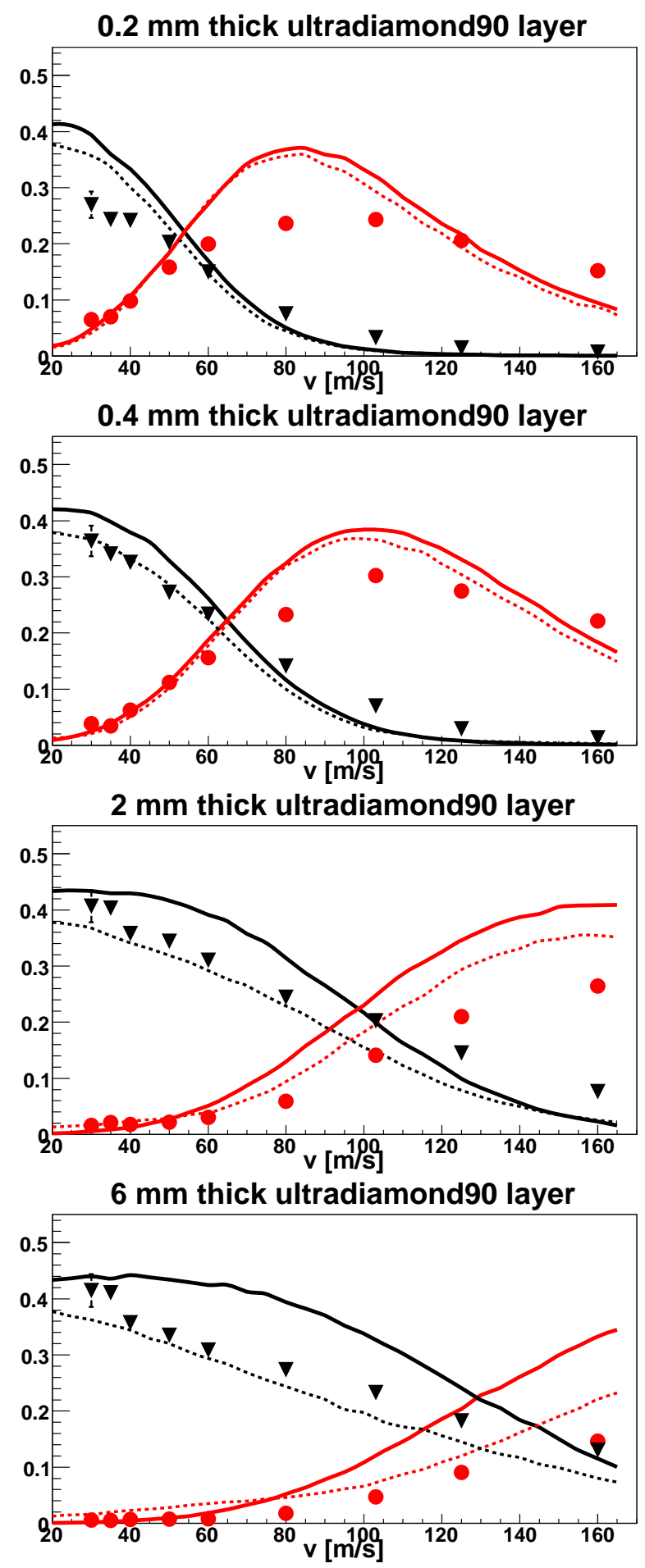

Fig. 4. The detection probability dependencies for backward (counters at $120^{\circ}+$ $150^{\circ}+210^{\circ}+240^{\circ}$ ) and forward (counters at $30^{\circ}+60^{\circ}+300^{\circ}+330^{\circ}$ ) scattering of $\mathrm{VCN}$ at the ultradiamond90 powder are shown as a function of VCN velocity, for different powder thicknesses. The triangles correspond to the measured probability of VCN backward scattering. The circles indicate the measured probability of VCN forward scattering. The solid lines correspond to the model of independent nanoparticles at rest. The dotted lines indicate the model that takes into account the up-scattering of $\mathrm{VCN}$ at water in the powder. 


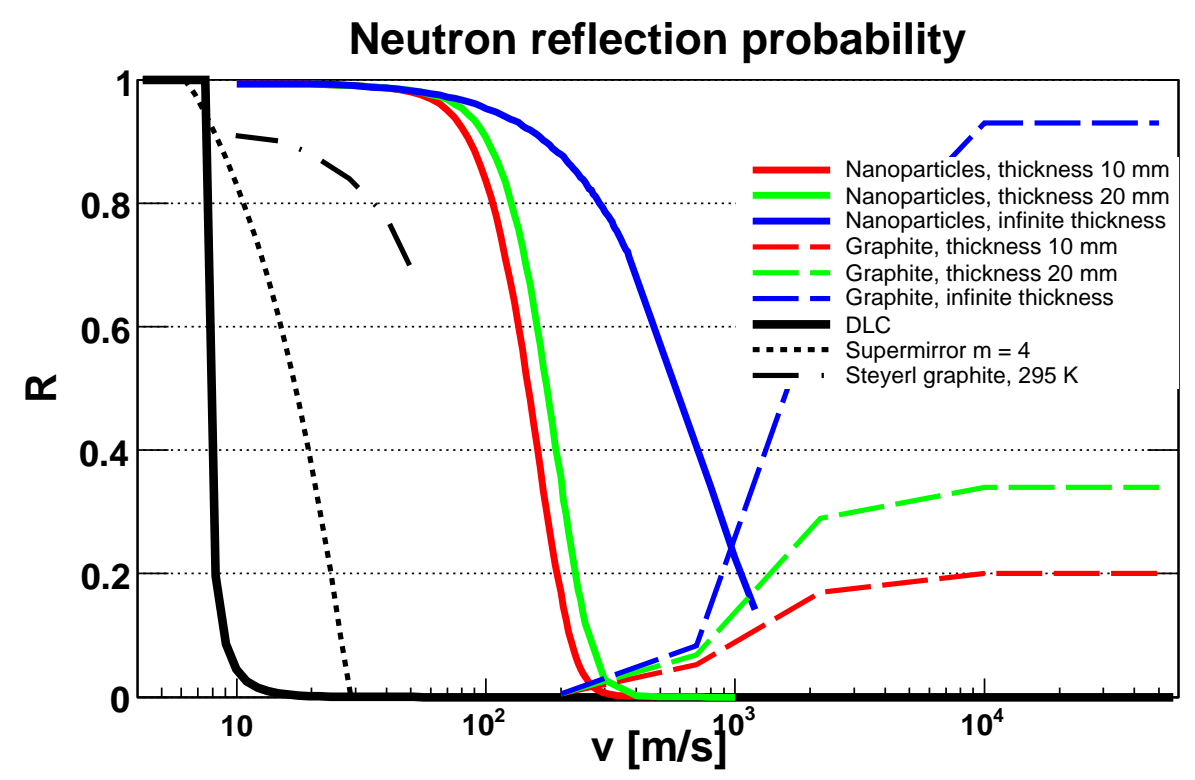

Fig. 5. The neutron reflection probability is shown as a function of neutron velocity for various carbon-based reflectors: 1) Diamond-like coating (DLC), with the highest known value of Fermi-potential (thick solid line) 2) The best present supermirror [27] (dotted line) 3) Steyerl's measurement of VCN reflection [21] (dased-dotted line) 4) Hydrogen-free ultradiamond90 powder for thicknesses of $10 \mathrm{~mm}, 20 \mathrm{~mm}$, and infinite (solid lines) 5) Reactor graphite reflectors at room temperature for thicknesses of $10 \mathrm{~mm}, 20 \mathrm{~mm}$, and infinite (dashed lines).

tion of scattered neutrons, and the predictions of the model of independent nanoparticles at rest. The hydrogen in the powder suppresses the penetration of neutrons across the samples more strongly than it decreases their reflection; this effect is more pronounced for small neutron velocities and for thick samples. In future studies we will remove the water and other hydrogen-containing impurities from the samples and cool the powder down to liquid nitrogen temperature (at which the upscattering of neutrons at hydrogen is largely suppressed). The residual discrepancy is probably due to poor knowledge of the spatial density distribution in the powder. This variation and even holes in the samples could affect the measurements, for the thinnest samples in particular.

As can be seen from fig. 4, the maximum energy of the reflected VCN and the reflection probability far exceed the corresponding values for the best supermirrors available [27], although the reflection is not specular at the nanopowder reflector of course.

If the reflection of the neutrons from the ultradiamond90 powder is determined uniquely by their scattering at the nanoparticles, and if we eliminate any additional losses (due in particular to hydrogen-containing impurities), the reflection probability would be as high as is shown in fig. 5. Efficient reflection would enable VCN to be stored in closed traps. We intend to explore this exciting hypothesis experimentally. 


\section{Conclusion}

We have observed extremely intense scattering of VCN at diamond powder nanoparticles for the first time. We have demonstrated that nanoparticle powders can be efficient reflectors of VCN with an energy value as high as $10^{-4} \mathrm{eV}$. This energy value and the reflection probability far exceed values for the best available supermirrors, although the reflection at nanoparticles is not specular. This phenomenon has a number of applications, including the storage of VCN in closed traps, reflectors for VCN and UCN sources, the more efficient guiding of $\mathrm{VCN}$ and, probably, of even faster neutrons.

\section{Aknowledgements}

We are grateful to Y. Calzavara, P. Geltenbort and C. Plonka for their assistance. This work is supported by RFBR grant number 03-02-16784-a.

\section{References}

[1] E. Fermi, "A course in neutron physics" in "Collected papers", The University of Chicago Press, Chicago (1965).

[2] R. Golub, D. Richardson and S.K. Lamoreaux, "Ultra-Cold Neutrons", Adam Higler (1991).

[3] V.K. Ignatovich, "The Physics of Ultracold Neutrons", Oxford University Press (1990).

[4] V.I. Luschikov, Yu.N. Pokotilovsky, A.V. Strelkov and F.L. Shapiro, JETP Lett. 9(1), 23-26 (1969).

[5] V.V. Nesvizhevsky, Phys. At. Nucl. 65, 400 (2002).

[6] P.J. De Carli and J.C. Jamieson, Science 133, 1821 (1961).

[7] http://www.ultradiamondtech.com

[8] A.E. Aleksenskii, M.V. Baidakova, A.Y. Vul', and V.I. Siklitskii, Physics of Solid State 41, 668 (1999).

[9] V.Yu. Dolmatov, Russian Chemistry Review 76, 339 (2007).

[10] E.V. Lychagin et al., Phys. At. Nucl. 65, 1995 (2002).

[11] D.G. Kartashov et al., IJN 6 (6), 501 (2007). 
[12] V.V. Nesvizhevsky, Phys. Usp. 46, 93 (2003).

[13] L.P. Mezhov-Deglin, V.V. Nesvizhevsky and A.V. Stepanov, Phys. Usp. 46, 89 (2003).

[14] L.P. Mezhov-Deglin et al., JLTP 148, 833 (2007).

[15] L.P. Mezhov-Deglin et al., JLTP 150 (3-4), 206 (2007).

[16] V.V. Nesvizhevsky, A.V. Strelkov, P. Geltenbort and P.S. Iaydjiev, Eur. J. Appl. Phys. 6, 151 (1999).

[17] V.V. Nesvizhevsky, A.V. Strelkov, P. Geltenbort and P.S. Iaydjiev, Phys. At. Nucl. 62, 776 (1999).

[18] A.V. Strelkov et al., NIM A440, 695 (2000).

[19] E.V. Lychagin et al., Phys. At. Nucl. 63, 548 (2000).

[20] V.V. Nesvizhevsky et al., Phys. Lett. B479, 353 (2000).

[21] A. Steyerl and W.-D. Trüstedt, Z.Physik 267, 379 (1974).

[22] S.S. Arzumanov et al., Phys. At. Nucl. 68, 1141 (2005).

[23] V.V. Nesvizhevsky, G. Pignol and K.V. Protasov, IJN 6 (6), 485 (2007).

[24] V.A. Artem'ev, Atomic Energy 101, 901 (2006).

[25] http://www.ill.fr/YellowBook/PF2

[26] A.L. Vereschagin, G.V. Sakovich, V.F. Komarov, and E.A. Petrov, Diamond and Related Materials 3, 160 (1993).

[27] R. Maruyama et al., Thin Solid Films 515, 5704 (2007). 


\section{Abstract}

Key words:

1

\section{References}

[] 